\title{
RASOnD - A comprehensive resource and search tool for RAS superfamily oncogenes from various species
}

\author{
Umay Kulsum ${ }^{\dagger}$, Vishwadeep Singh ${ }^{\dagger}$, Sujata Sharma, A Srinivasan, Tej P Singh and Punit Kaur ${ }^{*}$
}

\begin{abstract}
Background: The Ras superfamily plays an important role in the control of cell signalling and division. Mutations in the Ras genes convert them into active oncogenes. The Ras oncogenes form a major thrust of global cancer research as they are involved in the development and progression of tumors. This has resulted in the exponential growth of data on Ras superfamily across different public databases and in literature. However, no dedicated public resource is currently available for data mining and analysis on this family. The present database was developed to facilitate straightforward accession, retrieval and analysis of information available on Ras oncogenes from one particular site.

Description: We have developed the RAS Oncogene Database (RASOnD) as a comprehensive knowledgebase that provides integrated and curated information on a single platform for oncogenes of Ras superfamily. RASOnD encompasses exhaustive genomics and proteomics data existing across diverse publicly accessible databases. This resource presently includes overall 199,046 entries from 101 different species. It provides a search tool to generate information about their nucleotide and amino acid sequences, single nucleotide polymorphisms, chromosome positions, orthologies, motifs, structures, related pathways and associated diseases. We have implemented a number of user-friendly search interfaces and sequence analysis tools. At present the user can (i) browse the data (ii) search any field through a simple or advance search interface and (iii) perform a BLAST search and subsequently CLUSTALW multiple sequence alignment by selecting sequences of Ras oncogenes. The Generic gene browser, GBrowse, JMOL for structural visualization and TREEVIEW for phylograms have been integrated for clear perception of retrieved data. External links to related databases have been included in RASOnD.

Conclusions: This database is a resource and search tool dedicated to Ras oncogenes. It has utility to cancer biologists and cell molecular biologists as it is a ready source for research, identification and elucidation of the role of these oncogenes. The data generated can be used for understanding the relationship between the Ras oncogenes and their association with cancer. The database updated monthly is freely accessible online at http:// 202.141.47.181/rasond/ and http://www.aiims.edu/RAS.html.
\end{abstract}

\section{Background}

The driving force behind oncogenesis is the transformation of normal cells to uncontrolled cell proliferation and invasion. A number of genes involved in regulating the expression, growth and survival of cells have been identified in events leading to malignant transformation. Ras (RAt Sarcoma) is a major multigene superfamily

\footnotetext{
* Correspondence: punitkaur1@hotmail.com

† Contributed equally

Department of Biophysics, All India Institute of Medical Sciences, New Delhi110029, India
}

that has been implicated in approximately $30 \%$ of the known human tumors [1]. The predominant cancers involving Ras are associated with lung [2], colorectal region [3,4], pancreas [5] and thyroid [6]. The activation of Ras from a proto-oncogene into an oncogene results from a point mutation in the gene [7]. The Ras oncogenes cause hyperactive cell signalling and consequently contribute to the abnormal growth of the cell. These oncogenes have also been identified in human developmental disorders [8]. The Ras genes are expressed in nearly all tissues, though their expression levels may 
differ extensively. The most frequently observed Ras genes in human tumors are HRAS (Harvey-Ras), KRAS (Kristen-Ras) and NRAS (neuroblastoma-Ras) which vary in nature and specificity according to the cancer type [9].

The Ras proto-oncogene encodes a $21 \mathrm{kDa}(\mathrm{p} 21)$ small monomeric guanine nucleotide-binding protein. The Ras proteins play a central role in the control of normal and transformed cell proliferation. They have the ability to bind both guanosine triphoshate (GTP) and guanosine diphosphate (GDP) and function as a molecular switch in signal transduction by alternating between the inactive GDP-bound state and the active GTP-bound form. The Ras in the active form signals cell growth whereas in the inactive state it cannot initiate these pathways. This binary switch system of the Ras protein is localized to conformational changes in two distinct regions comprising switch I and switch II [10]. The structural changes in the mutated Ras hinder its ability to hydrolyse GTP. The molecular switch gets trapped in the 'switch on' state resulting in increased Ras-GTP levels. The signalling pathway is thus continuously stimulated which leads to oncogenesis [11]. The most commonly observed point mutations in human tumors are at codons $12 / 13$, or 61 . Mutations involving residues 59 , 63, 116, 117 and 119 have also been implicated in the oncogenic activation by Ras protein [12].

Ras was the first oncogene to be discovered among the transforming genes of the Harvey and Kirsten murine sarcoma viruses $[13,14]$. Since then a large number of Ras genes and proteins have been identified from different species including humans. On the basis of sequence similarity to the founding members [15], the Ras superfamily has been broadly classified into five main families, Ras [16], Rho [17], Arf [18], Rab [19] and Ran [20]. An additional family 'Others' has been assigned where their function is not clear. The Ras superfamily has grown and presently consists of over 150 members from humans [21]. Its orthologs have also been identified from nearly 100 other species. This superfamily has been the subject of several general [12,21-24] and specific reviews emphasizing their role in human and experimental cancer [25], cell signaling and transformation [26,27], cell motility [28], and differential functions in different tumors [29]. Most researchers across the globe have focused mainly on the identification, activation and clinical significance of these oncoproteins. This has led to the accumulation of enormous amount of data across numerous databases and in literature. There is no single database to our knowledge, presently available, where all information is contained on a single platform. This knowledgebase, compiles data from sequence to functional level accessible across diverse databases to enable the user rapid access, retrieval and analysis of information from one location. Thus, RASOnD aims to provide a better understanding of the Ras genes and proteins, their relationship with respect to each other and cancer.

\section{Construction and Content}

\section{Data Generation}

The database was developed with the objective to allow simple retrieval and exploratory analysis of information related to Ras oncogenes at a single point. It harbors a total of 199,046 entries from 101 species (Table 1). The details in RASOnD have been extracted from diverse public primary databases. The databases from which the information has been derived include the various resources available at National Centre for Biotechnology Information (NCBI) like GenBank [30] and Online Mendelian Inheritance in Man (OMIM), Kyoto Encyclopedia of Genes and Genomes (KEGG) [31], Database of Protein domains, families and functional sites (PROSITE) [32], Universal Protein Resource (UniProt) [33], Protein Databank (PDB) [34] and literature (PubMed). Various different keywords related to 'Ras' were used for extraction of data. The keywords combined with Ras included 'gene/oncogene', mutation, disease, expression, eukaryote and terms related to cancer like 'carcinoma/carcinogenesis/oncogenesis/tumor/hyperplasia/malignancy'. The search criteria also included expressions related to Ras like 'small monomeric G-proteins', 'GTP-binding proteins', 'small GTPases' and 'Ras related GTPases'. We have also annotated the data according to the associated cancer type as available in KEGG, OMIM and PubMed before April, 2011. Links to external databases have been included within each entry.

Table 1 Overview of the Information content in RASOnD

\begin{tabular}{cc}
\hline Data Type & Number \\
\hline Species & 101 \\
\hline Ras Families (excluding sub-families) & 6 \\
\hline Nucleotide FASTA Sequences & 4,882 \\
\hline Protein FASTA Sequences & 4,882 \\
\hline Single Nucleotide Polymorphisms (SNPs) & 102,426 \\
\hline Chromosome Positions & 1,033 \\
\hline Pfam Entries & 16,161 \\
\hline Prosite Entries & 1,656 \\
\hline Protein Profiles & 61,025 \\
\hline Protein Patterns & 4,900 \\
\hline PDB Structures & 548 \\
\hline Kegg Orthologies & 152 \\
\hline Kegg Entries & 3,871 \\
\hline Related Diseases & 30 \\
\hline Related Pathways & 3,626 \\
\hline
\end{tabular}




\section{Implementation Detail}

We have developed and implemented RASOnD using freely available online open source softwares. Apache HTTP Server serves as the localhost with MySQL relational database management system at the backend for storing and maintaining the data. The front end is designed with the help of HTML and CSS whereas the dynamic pages have been created through PHP. Parsing scripts written in PERL using regular expression were used to download data. The data is divided into 15 core tables with details on gene description, their family and sub-family, FASTA sequences - both nucleotide and protein, patterns, profiles, chromosome positions, related pathways, single nucleotide polymorphisms (SNPs), structures and links to related databases (Figure 1). The generic genome browser, GBrowse [35], open source molecular viewer JMOL [36] and TreeView [37] for phylograms have been implemented for effortless visualization and analysis. The user queries retrieve information from relational database tables which is displayed on the web interface. A local BLAST $[38,39]$ and CLUSTALW [40] tool can be used to retrieve and compare sequences of interest from within the database.

\section{Utility}

\section{User Interface - Simple and Advanced Search}

The interfaces in RASOnD are designed to facilitate straightforward navigation and exploitation of tools integrated in the database. The data stored in the RASOnD can be accessed employing a variety of search queries which allow the user to retrieve and analyze the data in a simple way. The users can retrieve information from the database through two user friendly interfaces comprising a 'Simple' and an 'Advanced' search. A search through the database can be performed separately on a particular feature in the simple search as well as simultaneously on multiple fields using the advanced search option. The simple search allows the user to explore the database by selecting a specific individual field from the drop down menu. The user can perform a search by entering the keyword or by selecting the options available in the interface. Various alternatives are present in the simple search.

The "gene name or INSD (International Nucleotide Sequence Database) accession number" can be input by the user to extract all available information on a particular Ras oncogene (Figure 2). The "gene family" option allows the investigator to select a family from the five main families of Ras superfamily. The option of further selecting a member from the subfamilies of Ras, Rab, Rho, Ran, Arf/Sar family and Others is also incorporated. The information on all Ras oncogenes present in a specific species can be retrieved by choosing the "organism search" option from the dropdown menu.
The "motif search" allows the user to scan motifs related to a gene. This comprises a pattern and a profile search which generates data on exisiting patterns and predicted motifs respectively obtained from PROSITE scan. The user can also opt for the pathway of interest where these oncogenes are involved via the "pathway search" menu. The "chromosome position" alternative permits the user to access the details for genes present in a desired organism. Selection of the desired organism will present a table pertaining to the chromosome number and position and related KEGG and NCBI entry.

We have incorporated the "Advance search" option to allow search of multiple fields simultaneously. This search includes all the fields available in the simple search menu. The user in this interface can select specific features and thus restrict the field of search. The user can (i) input the gene or explore all genes based on the subsequent parameters (ii) use the different aspects incorporated as a drop down menu to access all or one entry from the various parameters like organism, Ras family, related pathways, patterns, profiles and disease and (iii) decide to include or exclude the protein structures and SNPs. Thus, the user can narrow down the search to the requisite criterion by utilizing this interface (Figure 3). The initial output from this interface displays the user selection before providing the tabulated results.

\section{User Interface - Diseases}

The Ras oncogenes have been implicated in a variety of cancers and developmental disorders. The human cancers which have the presence of Ras oncogenes are included as a dropdown menu under the 'Disease' section. The user can choose the disease of interest from this menu. On selection, initially a summary table on human Ras members involved in the preferred disease is returned with a 'Browse' button. The related Ras oncogenes/proteins from other species are also indicated. This page also furnishes a short description of the cancer type.

\section{User Interface - Results}

The results for each search are displayed in two parts. An initial investigation using any of the search options returns a summary table displayed in tabular format listing the Ras oncogenes from different species with a 'Browse' button. A click on this button presents additional and detailed records on varied aspects of the selected oncogene like gene name with the nucleotide and protein sequences, KEGG orthology, pathways, chromosome position, available structures, motifs and SNPs for all the genes belonging to the selected organism (Figure 4). The diseases with Ras manifestation are also exhibited which have been further hyperlinked to KEGG. Users can graphically view the motifs via the 


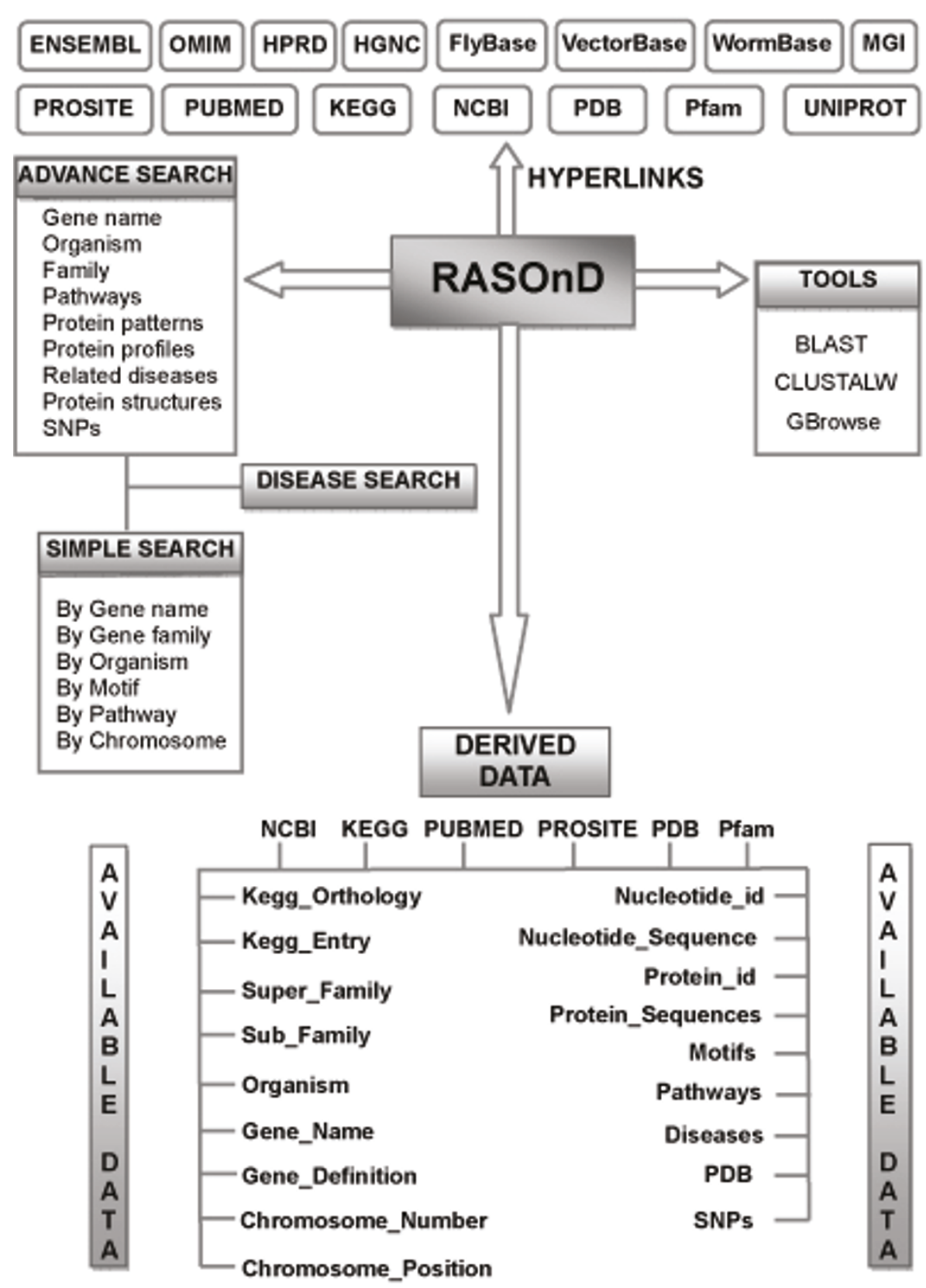

Figure 1 RASOnD Database scheme. The conceptual scheme depicting the various components of the database.

GBrowse tool. The PDB Ids have been reported where the three-dimensional structure is available. The coordinates can be downloaded or structures analysed with the JMOL applet embedded in the database. A hyperlink to RCSB Protein Data Bank has also been integrated. Similarly, the nucleotide or protein sequence data at each point can be retrieved or a local BLAST run to obtain sequences similar to the one of interest. The homologous sequences returned from the BLAST search can be further selected for onward submission to
CLUSTALW for multiple sequence alignment. The selection can be made via a click on the buttons provided on the edge of the sequence. We have also hyperlinked each entry to the related databases like HPRD, KEGG, OMIM and HGNC. In the Pathways page the results are presented as KEGG Pathway ID, related organism, KEGG Entry and pathway image which can be further chosen for obtaining detailed information on the oncogene. Links to the KEGG database are included for scrutiny of proteins implicated in that pathway. 


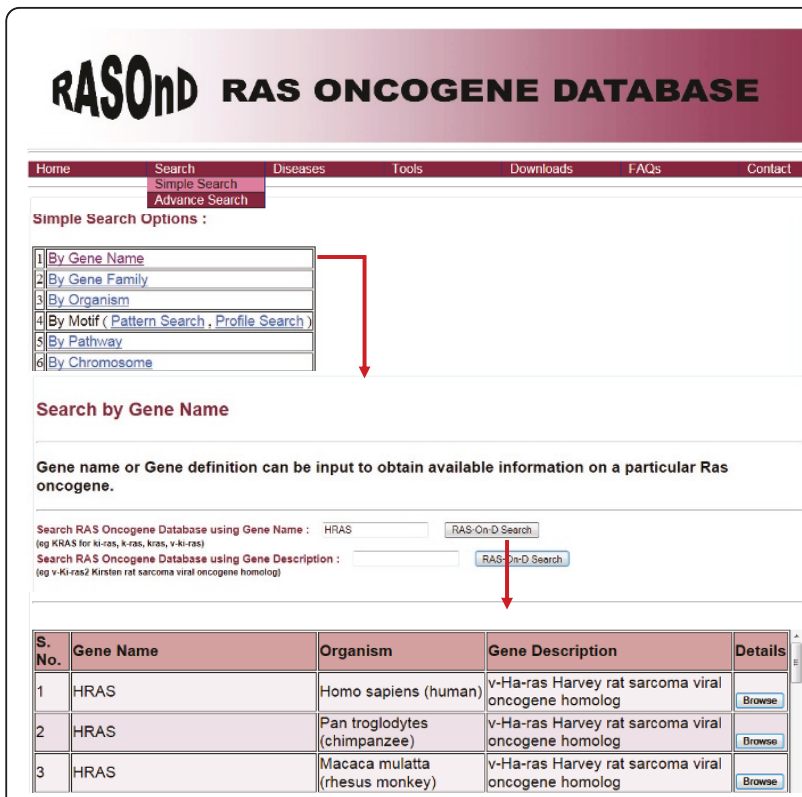

Figure 2 Screen shots of User Interface - Simple Search. Simple search query using one of the six options provided e.g. Gene Name. This query on providing the gene name results in a page containing the organism name, the gene description and a 'Browse' button. A click on this button provides details related to that particular gene. The red arrows indicate the pages obtained on clicking the 'Submit' button

\section{WebTools}

The analysis of the data within the database can be carried out using the tools available in the database. Three tools have been implemented. The users can utilize the genome viewer, 'GBrowse' for browsing associated genes and patterns and profiles of predicted motifs for the selected oncogene. The user can carry out sequence similarity searches from within the database using 'RASON-DBLAST'. The user-defined query nucleotide or protein sequence in FASTA format can be submitted for a local BLAST search against the database to identify homologous sequences. The default or user-defined parameters like e-value cutoff, gap opening, word size or matrix can be modified for such a search. The user can choose sequences returned for direct onward submission to CLUSTALW or download the selected sequences for any other analysis. "CLUSTALW" can be used for the multiple sequence alignment of the selected sequences obtained from the database query. The user can also enter additional sequences of interest. The phylograms generated from CLUSTALW tool can be observed with TreeView.

\section{Additional features}

The home page includes a FAQ page with the purpose of providing basic facts on the Ras superfamily as well as the usage of the entire database. This page also includes a section which is hyperlinked to reviews

\section{RASOnd RAs Oncogene database}

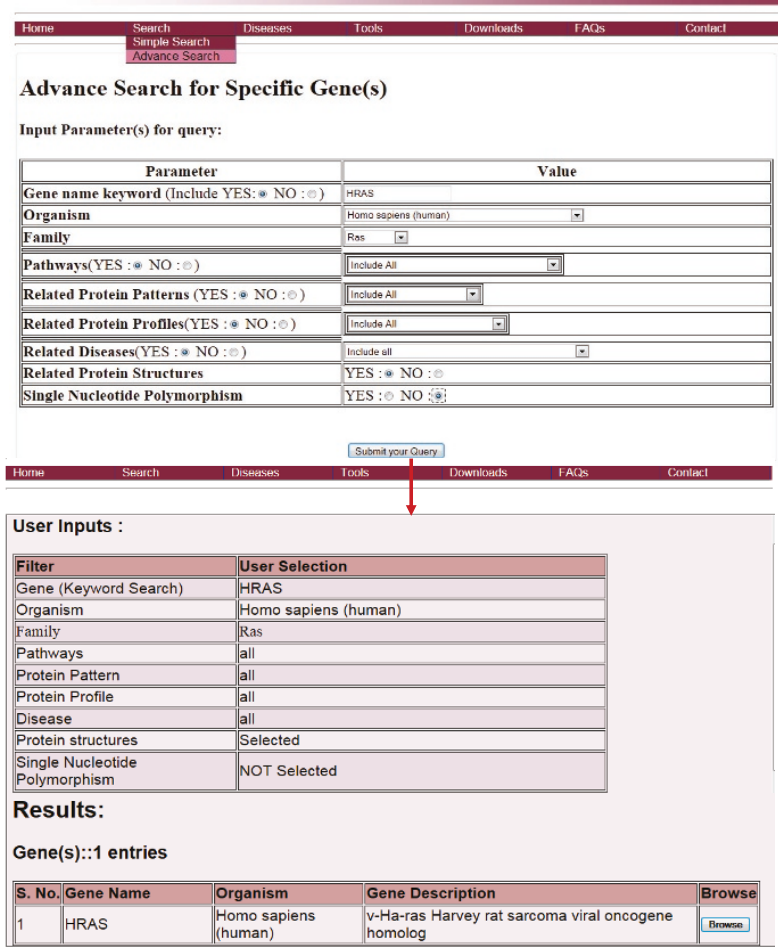

Figure 3 Screen shots of User Interface - Advanced Search. The advanced search interface where more than one option can be selected simultaneously to obtain varied information about the Ras gene/protein. The user can choose to include or exclude any of the options provided. The user has to input the gene name of interest and then further pick either a parameter from the drop down menu or decide on one of the default buttons incorporated for the search. Submission of this option returns the genes according to the search criteria. A click on the 'Browse' button will return detailed data on the selected gene.

available in literature. A download option has been provided to obtain data related to nucleotide sequences, protein sequences, single nucleotide polymorphisms and protein structures as separate files. Links to other databases which refer to some Ras superfamily members and latest available literature from PubMed have also been included on the home page.

\section{Discussion}

The main focus of research in molecular cancer is not only the identification of the genes altered in different tumor types but also determination of the pathological role played by them in tumorogenesis. The Ras genes and proteins are particularly very significant as they are activated by point mutations and are the predominant oncogenes in human tumors. The comprehension of different single amino acid substitutions which lead to 


\section{rASOnd ras Oncogene database}

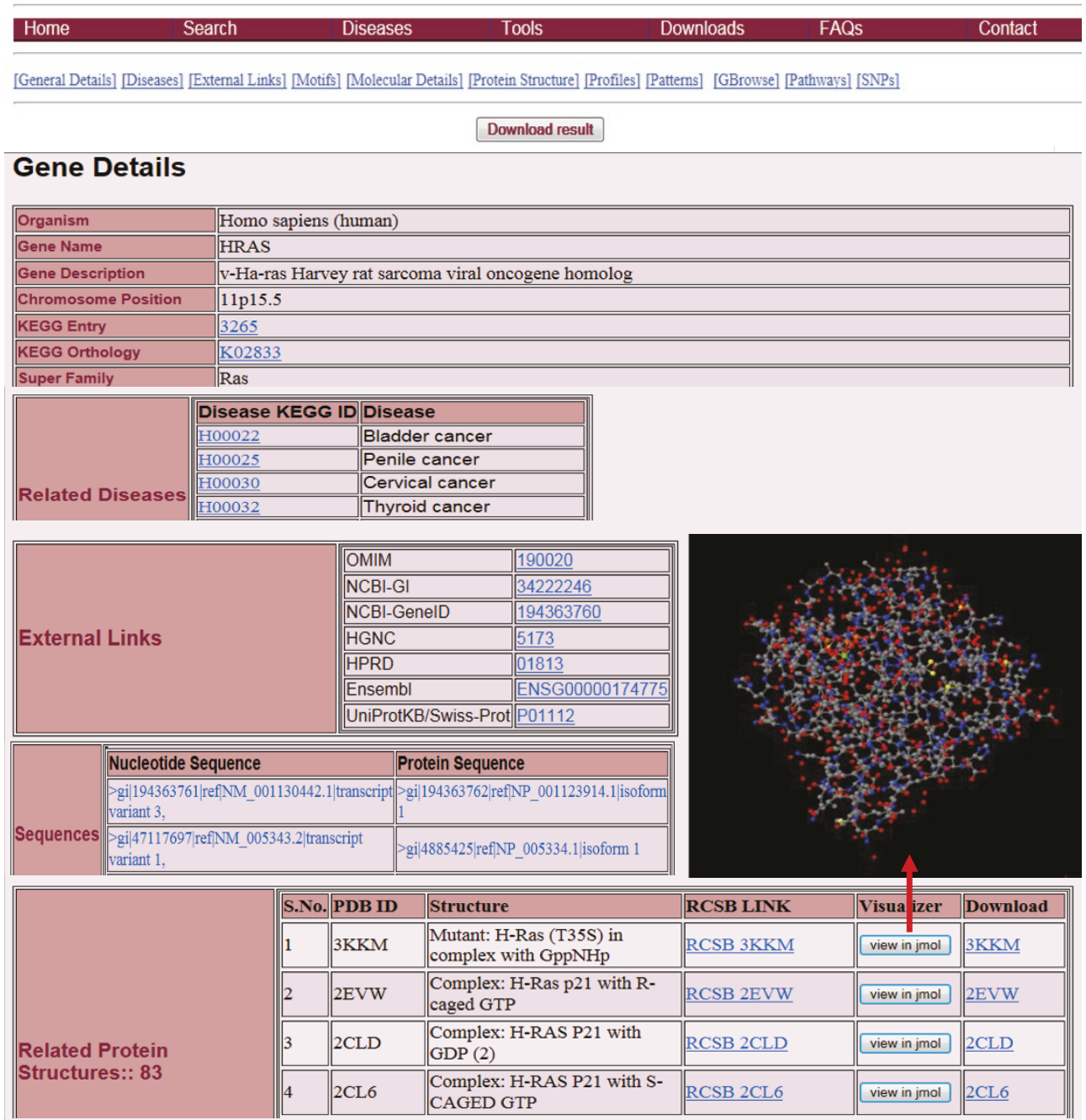

GBrowse: Refer to FAQ for usage.

View in larger window

\begin{tabular}{|c|c|c|}
\hline \multicolumn{3}{|l|}{ Examples: 34222246.} \\
\hline \multicolumn{3}{|c|}{$\begin{array}{l}\text { [Bookmark this] [Upload your own data] [Hide banner] [Share these tracks] [Link to Image] [High-res Image] [Help] [Rकs] } \\
\text { [Search }\end{array}$} \\
\hline Landmark or Region: & & Reports \& Analysis: \\
\hline 34222246 & Search & Download Decorated FASTA File - Configure... \\
\hline Overview & & \\
\hline
\end{tabular}

Figure 4 Screen shots of User Interface - Result Page. Collage of some of the results produced via the 'Browse' button in both 'Simple' and 'Advanced' search options.

malignancy is increasingly becoming an important tool to elucidate the mechanisms of oncogenesis. Moreover, the Ras dependent pathways are now being targeted for the development of anti-cancer agents. The objective of this database is to amalgamate information distributed across diverse platforms to a single site. It stores data related to Ras genes and proteins, related polymorphisms, their pathways, associated diseases, motifs and structures. It also contains tools for the examination and analysis of these sequences. It thus, serves as an 
organized source to both clinicians and basic scientific community for research on Ras superfamily. This resource has been designed to allow the user to explore and extract effortlessly all accessible facts at one common place. RASOnD attempts to bridge the gap between the genomics and system biology and affords inputs and links to all aspects of Ras oncogenes not accessible as a central resource so far. RASOnD is of interest to molecular oncologists and researchers working in the field of cancer as it brings under one roof, data for which otherwise a search would have to be conducted across varied resources. It, therefore, serves as a useful platform for ready reference for identification, determination and comparative analysis of the genes and proteins belonging to the Ras superfamily.

\section{Comparison to other related Databases}

There is no similar database to RASOnD, however, there exist a small number of databases which have some reference to the 'Ras' gene/protein "see Additional file 1, Figure S1". These deal with only a few species (unlike the present which includes information on all available species) or present specific rather than generalized and detailed information on the 'Ras' superfamily. Majority have a focus on genomics rather than a proteomics approach unlike RASOnD which provides detailed information on both aspects. These databases include ACTuDB [41], Catalog of Somatic Mutations in Cancer (COSMIC) database [42], Dragon database for exploration of ovarian cancer genes (DDOC) [43], kinase pathway database [44], mouse genome database [45], Oncogenomic Database of Hepatocellular carcinoma (OncoDB.HCC) [46], rat genome database (RGD) [47], Signal Transduction Classification (STCDB) [48], Tumor gene family of databases (TGDB) [49] and Genecards [50]. The rat and mouse genome databases are useful if the focus of search is on these species. COSMIC database contains information on published somatic mutations only in various cancers and refers to just four families of Ras in humans. The DDOC and OncoDB.HCC resources are specific to Ras members implicated in ovarian cancer and hepatocellular carcinoma respectively. The DDOC deals with human Ras members whereas OncoDB.HCC comprises details on rat and mouse besides human members. The ACTuDB contains information on the genomic profiling of tumors only with little emphasis on Ras oncogenes while STCDB has reference to a handful of Ras genes/ proteins implicated in signal transduction. The Kinase pathway database refers to only seven species. The GeneCards contains the maximum number of Ras members after RASOnD, however, it also refers to only some species. A link to these databases is available on the home page.
RASOnD is distinct from these published on-line public databases. The main differentiating feature is the inclusion of genomics and proteomics data from all species where Ras members have been identified. Thus, it is the single, specialized largest repository of Ras oncogenes. The purpose of developing RASOnD was to provide a simple solution to clinicians and oncologists to extract information on this gene. Moreover, it affords the alternative of selecting the disease of interest rather than just the Ras oncogene. The initial exploratory sequence analysis from BLAST can further be exploited for multiple sequence alignment with CLUSTALW by a one step selection of the returned sequences. This is a feature unique to the present database. In this manner, the concept of RASOnD is different from other available databases.

\section{Future Directions}

The present database focuses mainly on the genes and proteins included in the Ras superfamily, their integration and involvement in different tumors and provides tools for their analysis. We further plan to extend the database with a greater emphasis on the Ras proteins to include their post-translation modifications, interacting partners and inhibitors. Proteins containing the RASbinding domain will also be incorporated into the database.

\section{Conclusion}

The RASOnD knowledgebase is the first attempt to construct an easily accessible and handy platform on the multifaceted large Ras superfamily. RASOnD presents the end-user with all possible details for analysis and retrieval on various aspects of RAS oncogenes in a simple and user friendly manner. Details about various other databases with reference to the Ras have been included. The information in the database can be accessed and investigated by a single query search or by a combination of various queries. Alternatively, the researcher can determine the Ras oncogene involved in a particular cancer type by exercising the dropdown menu under diseases. JMOL, GBrowse and TreeView have been implemented for easy visualization whereas BLAST and CLUSTALW modules have been incorporated for comparative analysis of sequences within the database. The wealth of information available in RASOnD can thus be exploited by both bench side workers and bioinformaticians to carry out a comprehensive analysis on the Ras superfamily. The computational biologists can utilize the compiled data to develop computational prediction tools for novel mutations.

\section{Availability and Requirements}

The RASonD database will be continuously updated and upgraded and is freely available at http://www.aiims.edu/ 
RAS.html and http://202.141.47.181/rasond/ to academic and non-academic users. Java enabled client web browser is required for the usage of the tools GBrowse, Jmol applet and Treeview.

\section{Additional material}

\section{Additional file 1: Figure S1 - Comparison to other related}

databases. A three-dimensional bar plot indicating the comparison of 'Ras - related' information incorporated in RASOnD with other related resources.

\section{Acknowledgements}

The work was supported by Indian Council of Medical Research in the form of the Bio-Medical Informatics Centre (Grant No. P\&l/BIC/1/1/2009). The assistance provided by Mr. Harishankar is also gratefully acknowledged.

\section{Authors' contributions}

UK and VS collected and compiled the database from public databases and literature and developed the database. AS refined the manuscript. SS and TPS coordinated the project. PK conceived and designed this study and prepared the manuscript. All authors have read and approved the final manuscript.

\section{Competing interests}

The authors declare that they have no competing interests.

Received: 1 April 2011 Accepted: 5 July 2011 Published: 5 July 2011

\section{References}

1. Malumbres A, Barbacid M: To cycle or not to cycle: a critical decision in cancer. Nat Cancer Rev 2001, 1:222-235.

2. Chiosea S, Shuai Y, Cieply K, Nikiforova MN, Dacic S: EGFR fluorescence in situ hybridization-positive lung adenocarcinoma: incidence of coexisting KRAS and BRAF mutations. Hum Pathol 2010, 41:1053-1060.

3. Prenen $H$, Tejpar S, Van Cutsem E: New strategies for treatment of KRAS mutant metastatic colorectal cancer. Clin Cancer Res 2010, 16:2921-2926.

4. Andreyev HJ, Norman AR, Cunningham D, Oates J, Dix BR, lacopetta BJ, Young J, Walsh T, Ward R, Hawkins N, Beranek M, Jandik P, Benamouzig R, Jullian $E$, Laurent-Puig $P$, et al: Kirsten ras mutations in patients with colorectal cancer: The 'RASCAL II' study. Br J Cancer 2001, 85:692-696.

5. Abou-Alfa GK, Chapman PB, Feilchenfeldt J, Brennan MF, Capanu M, Gansukh B, Jacobs G, Levin A, Neville D, Kelsen DP, O'reilly EM: Targeting Mutated K-ras in Pancreatic Adenocarcinoma Using an Adjuvant Vaccine. Am J Clin Oncol 2010.

6. Levy R, Grafi-Cohen M, Kraiem Z, Kloog Y: Galectin-3 Promotes Chronic Activation of K-Ras and Differentiation Block in Malignant Thyroid Carcinomas. Mol Cancer Ther 2010, 9:2208-2219.

7. Tabin CJ, Bradley SM, Bargmann Cl, Weinberg RA, Papageorge AG, Scolnick EM, Dhar R, Lowy DR, Chang EH: Mechanism of activation of a human oncogene. Nature 1982, 300:143-149.

8. Rauen KA, Schoyer L, McCormick F, Lin AE, Allanson JE, Stevenson DA, Gripp KW, Neri G, Carey JC, Legius E, Tartaglia M, Schubbert S, Roberts AE, Gelb BD, Shannon K, et al: Proceedings from the 2009 genetic syndromes of the Ras/MAPK pathway: From bedside to bench and back. Am J Med Genet A 2010, 152A:4-24.

9. Lowy DR, Willumsen BM: Function and regulation of ras. Annu Rev Biochem 1993, 62:851-891.

10. Wittinghofer A, Waldmann H: Ras-A Molecular Switch Involved in Tumor Formation. Angewandte Chemie 2000, 39:4192-4214.

11. Konstantinopoulos PA, Karamouzis MV, Papavassiliou AG: Post-translational modifications and regulation of the RAS superfamily of GTPases as anticancer targets. Nat Rev Drug Discov 2007, 6:541-555.

12. Trahey M, McCormick F: A cytoplasmic protein stimulates normal N-Ras p21 GTPase, but does not affect oncogenic mutants. Science 1987, 238:542-545.
13. Harvey JJ: An unidentified virus which causes the rapid production of tumors in mice. Nature 1964, 204:1104-1105.

14. Kirsten WH, Mayer LA: Morphologic responses to a murine erythroblastosis virus. J Natl Cancer Inst 1967, 39:311-335.

15. Wennerberg K, Rossman KL, Der CJ: The Ras superfamily at a glance. Journal of Cell Science 2005, 118:843-846.

16. Reuther GW, Der CJ: The Ras branch of small GTPases:Ras family members don't fall far from the tree. Curr Opin Cell Biol 2000, 12:157-165.

17. Sahai E, Marshall CJ: Rho-GTPases and cancer. Nat Rev Cancer 2002, 2:133-142.

18. Donaldson JG, Jackson CL: Regulators and effectors of the ARF GTPases. Curr Opin Cell Biol 2000, 12:475-482.

19. Samantha L, Schwartz CC, Pylypenko O, Rak A, Wandinger-Ness A: Rab GTPases at a glance. Journal of Cell Science 2007, 120:3905-3910.

20. Kuersten S, Ohno M, Mattaj IW: Nucleocytoplasmic transport: ran, beta and beyond. Trends Cell Biol 2001, 11:497-503.S.

21. Macara IG, Lounsbury KM, Richards SA, McKiernan C, Bar-Sagi D: The Ras superfamily of GTPases. FASEB J 1996, 10:625-630.

22. Goodsell DS: The molecular perspective: the ras oncogene. Oncologist 1999, 4:263-264.

23. Malumbres M, Barbacid M: RAS oncogenes: the first 30 years. Nature Reviews Cancer 2003, 3:459-465.

24. Barbacid M: ras genes. Ann Rev Biochem 1987, 56:779-827.

25. Roberts PJ, Der CJ: Targeting the Raf-MEK-ERK mitogen-activated protein kinase cascade for the treatment of cancer. Oncogene 2007, 26:3291-3310.

26. Malumbres M, Pellicer A: Ras pathways to cell cycle control and cell transformation. Frontiers in Bioscience 1998, 3:d887-d912.

27. Mitin N, Rossman KL, Der CJ: Signaling interplay in Ras superfamily function. Curr Biol 2005, 15:R563-R574.

28. Oxford G, Theodorescu D: Ras superfamily monomeric $\mathrm{G}$ proteins in carcinoma cell motility. Cancer Letters 2003, 189:117-128.

29. Moon A: Differential functions of Ras for Malignant phenotypic conversion. Arch Pharm Res 2006, 29:113-122.

30. Benson DA, Karsch-Mizrachi I, Lipman DJ, Ostell J, Wheeler DL: GenBank. Nucleic Acids Res 2008, 36:D25-D30.

31. Kanehisa M, Goto S, Furumichi M, Tanabe M, Hirakawa M: KEGG for representation and analysis of molecular networks involving diseases and drugs. Nucleic Acids Res 2010, 38:D355-D360.

32. Sigrist CJA, Cerutti L, de Castro E, Langendijk-Genevaux PS, Bulliard V, Bairoch A, Hulo N: PROSITE, a protein domain database for functional characterization and annotation. Nucleic Acids Res 2010, 38:161-166.

33. UniProt Consortium: The universal protein resource (UniProt). Nucleic Acids Res 2008, 36:D190-D195.

34. Kouranov A, Xie L, de la Cruz J, Chen L, Westbrook J, Bourne PE, Berman HM: The RCSB PDB information portal for structural genomics. Nucleic Acids Res 2006, 34:D302-D305.

35. Stein LD, Mungall C, Shu S, Caudy M, Mangone M, Day A, Nickerson E, Stajich JE, Harris TW, Arva A, Lewis S: The generic genome browser: a building block for a model organism system database. Genome Res 2002, 12:1599-1610.

36. Jmol: an open-source Java viewer for chemical structures in 3D. [http:// wWw.jmol.org/].

37. Page RDM: TREEVIEW: An application to display phylogenetic trees on personal computers. Computer Applications in the Biosciences 1996, 12:357-358.

38. Altschul SF, Gish W, Miller W, Myers EW, Lipman DJ: Basic local alignment search tool. J Mol Biol 1990, 215:403-410.

39. Johnson M, Zaretskaya I, Raytselis Y, Merezhuk Y, McGinnis S, Madden TL: NCBI BLAST: a better web interface. Nucleic Acids Res 2008, , 36: W5-W9.

40. Larkin MA, Blackshields G, Brown NP, Chenna R, McGettigan PA, McWilliam H, Valentin F, Wallace IM, Wilm A, Lopez R, Thompson JD, Gibson TJ, Higgins DG: ClustalW and ClustalX version 2. Bioinformatics 2007, 23:2947-2948.

41. Hupé P, La Rosa P, Liva S, Lair S, Servant N, Barillot E: ACTuDB, a new database for the integrated analysis of array-CGH and clinical data for tumors. Oncogene 2007, 26:6641-6652 [http://bioinfo-out.curie.fr/actudb/].

42. Forbes SA, Bhamra G, Bamford S, Dawson E, Kok C, Clements J, Menzies A, Teague JW, Futreal PA, Stratton MR: The Catalogue of Somatic Mutations in Cancer (COSMIC). Curr Protoc Hum Genet 2008, Chapter 10: [http://www. sanger.ac.uk/genetics/CGP/cosmic/, Unit 10.11.

43. Kaur M, Radovanovic A, Essack M, Schaefer U, Maqungo M, Kibler T, Schmeier S, Christoffels A, Narasimhan K, Choolani M, Bajic VB: Dragon 
database for exploration of functional context of genes implicated in ovarian cancer. Nucleic Acids Res 2009, 37:D820-D823 [http://apps.sanbi.ac. za/ddoc/index.php].

44. Koike A, Kobayashi Y, Takagi T: Kinase pathway database: an integrated protein-kinase and NLP-based protein-interaction resource. Genome Res 2003, 13:1231-1243.

45. Bult CJ, Kadin JA, Richardson JE, Blake JA, Eppig JT: The Mouse Genome Database: enhancements and updates. Nucleic Acids Res 2010, 38 : D586-D592 [http://www.informatics.jax.org/]

46. Su WH, Chao CC, Yeh SH, Chen DS, Chen PJ, Jou YS: OncoDB.HCC: an integrated oncogenomic database of hepatocellular carcinoma revealed aberrant cancer target genes and loci. Nucleic Acids Res 2007, 35: D727-D731 [http://oncodb.hcc.ibms.sinica.edu.tw/index.htm].

47. Twigger S, Lu J, Shimoyama M, Chen D, Pasko D, Long H, Ginster J, Chen CF, Nigam R, Kwitek A, Eppig A, Maltais A, Maglott D, Schuler G, Jacob H, Tonellato PJ: Rat Genome Database (RGD): mapping disease onto the genome. Nucleic Acids Res 2002, 30:125-128 [http://rgd.mcw.edu/].

48. Chen M, Lin S, Hofestaedt R: STCDB: Signal Transduction Classification Database. Nucleic Acids Res 2004, 32:D456-D458 [http://bibiserv.techfak.unibielefeld.de/stcdb/].

49. Tumor Gene family of Databases (TGDB). [http://www.tumor-gene.org/ TGDB/tgdb.html].

50. Safran M, Dalah I, Alexander J, Rosen N, Iny Stein T, Shmoish M, Nativ N, Bahir I, Doniger T, Krug H, Sirota-Madi A, Olender T, Golan Y, Stelzer G, Harel A, Lancet D: GeneCards Version 3: the human gene integrator. Database 2010 [http://www.genecards.org/], baq020.

doi:10.1186/1471-2164-12-341

Cite this article as: Kulsum et al.: RASOnD - A comprehensive resource and search tool for RAS superfamily oncogenes from various species. BMC Genomics 2011 12:341.

\section{Submit your next manuscript to BioMed Central and take full advantage of:}

- Convenient online submission

- Thorough peer review

- No space constraints or color figure charges

- Immediate publication on acceptance

- Inclusion in PubMed, CAS, Scopus and Google Scholar

- Research which is freely available for redistribution

Submit your manuscript at www.biomedcentral.com/submit 